\title{
Wireless Regional Access Networks: A Wise Choice for Internet Connectivity to Rural Areas of Zimbabwe
}

\author{
Mardeni R. ${ }^{1} \&$ Chimheno R. L. ${ }^{1}$ \\ ${ }^{1}$ Multimedia University, Jalan Multimedia, Cyberjaya Selangor, Malaysia \\ Correspondence: Mardeni R., Multimedia University, Jalan Multimedia 63100, Cyberjaya Selangor, Malaysia. \\ Tel: 60-3-8312-5481. E-mail: mardeni.roslee@mmu.edu.my
}

\author{
Received: September 25, $2012 \quad$ Accepted: May 3, $2013 \quad$ Online Published: June 18, 2013 \\ doi:10.5539/mas.v7n7p29 URL: http://dx.doi.org/10.5539/mas.v7n7p29
}

\begin{abstract}
Wireless Regional Access Network (WRAN) is increasingly becoming the focus architecture for future rural and remote area access networks. Firstly, we explain "the Zimbabwean problem", and then give brief discussions of existing technologies deployed for rural communications elsewhere in the world. As we searched for solutions to the Zimbabwean problem, special attention was given to technologies such as cognitive radio networks. In the process, WRAN, which saves on deployment and license costs, has extended coverage range, increased cell capacity and utilises idle TV spectrum, was identified as a suitable broadband access candidate for providing connectivity to the rural areas of Zimbabwe. A design network structure suitable for rural areas in Zimbabwe is proposed and a basic cognitive radio (CR) simulation to show the possibility of harnessing idle Zimbabwe Broadcasting Corporation TV channels/frequency bands using CR technology was successfully run. Power spectrum density estimation model for sensing was used. A number of implementation challenges such as the effect of antenna height on the hidden terminal problem, MIMO incorporation in order to mitigate for such effects in WRAN networks, the investigation and mitigation of interference contribution from remote television stations, WRAN networks and other primary stations and users are discussed.
\end{abstract}

Keywords: MIMO, broadband wireless access, WRAN, CR simulation, rural areas, zimbabwean-problem, cognitive radio networks

\section{Introduction}

According to recent International Telecommunication Union (ITU) reports $(2010,2011)$, about $7.8 \%$ of Zimbabweans have access to the internet. This has been attributed to a number of reasons collectively known as "the Zimbabwean problem" which include limited bandwidth problems, high cost of services, and inefficiency in existing broadband networks and to a greater extend - no internet coverage in rural areas, rural schools and small village towns. These areas usually referred to as growth points, where the majority of Zimbabweans reside. These issues and many others including inefficiencies in existing broadband networks, limited the expansion of network access to the rural schools and growth points. Hence new players with innovative low cost internet connectivity would find a ready market of both existing Internet users and new subscribers (http://www.techzim.co.zw/2010/10/no-real-broadband-yet-in-zimbabwe/). The above mentioned problems prompted this project. This project will help minimise and eventually eradicate the digital bias that exists between urban and rural communities. Other benefits of this project apart from solving the Zimbabwean problem include the uplifting of the community's educational and economic standard through e-learning and e-banking etc. CR technology being a new technology has very few simulation packages. The few packages available simulate only targeted aspects of cognitive radio systems such as MIMO techniques and interference in CR networks. Basically simulators that were suitable for the demand of cognitive radio simulations could not be found. What was apparent was that many researchers implemented their algorithms for cognitive radios on network simulators such as NS-2, OPNET, and QUALNET which were created for the ordinary wireless network. These were found to be very difficult to implement CR algorithms. Only one simulator which was implemented in MATLAB by the authors of http://intcube.com/edu/projects/codes/crad.m was found. This is the simulator that we modified. The beauty of the proposed simulation is that it is simple, easy to follow and implement and yet produce results that emulate real time processes. This project therefore, provides an improved but easy to understand simulation model in MATLAB for the study and implementation of WRAN networks. 
We also provide an easy to follow flow chart which incorporates TV broadcasting time slots and can be modified easily for improvements of the algorithms for CR Networks. A lot of research work has already been done in connection with cognitive radio networks for rural areas. The most promising cognitive radio technology for rural areas is IEEE 802.22 which is being developed to utilise the opportunistic access of the TV frequencies (Liang, Hoang, \& Chen, 2008). This standard which caters for cognitive radio based WRAN was suggested so as to enable rural broadband wireless access using cognitive radio technology in TV whitespaces. The existence of idle TV bands in Zimbabwe made this kind of cognitive radio technology more desirable and possible. Recent survey by Zimbabwe All Media Products, in television viewership shows that more than 60 per cent of Zimbabweans who own televisions have abandoned the state controlled Zimbabwe Broadcasting Corporation Television (ZBC TV) in favour of free to air regional and international channels (http://www.thestandard.co.zw/local/28597-zbc-tv-viewership-hits-rock-bottom.html). The rest of the project is organized as follows: A brief discussion of existing technologies proposed or deployed for rural communications in other countries with similar internet access problems; Relevant reviews of recent developments in MIMO and Cognitive Radio Technologies in general with special emphasis on Cognitive Radio in idle TV Bands; A design proposal of a broadband access network using IEEE 802.22 cognitive radio technology proposal for Zimbabwean rural communities; Implementation challenges, followed by the Conclusions and Future studies.

\section{Existing Technologies}

There are numerous documented research papers on possible ways of providing internet connectivity to rural areas using existing technologies. These technologies deployed include the use of: Satellite, Cellular, Wi-Fi, WIMAX and Hybrid Broadband Access networks to name just a few. These technologies have their pros and cons but the major drawback for all of them hinges on the affordability of the end user to take up the service; for the rural market these networks would be unviable hence unsuitable for rural areas. However new ways continue to be sought in order to bridge the financial gap using combinations of these existing technologies e.g. use of Wireless Mesh Networks.

\section{Relevant Literature Review}

MIMO TECHNOLOGY: In general, multiple-input multiple-output (MIMO) antenna technology has advanced in leaps and bounds and has becomes a key player, in wireless networks for achieving the required high spectral efficiency (Bauch \& Alexiou, 2008). MIMO's inherent ability for high capacity, enhanced diversity, and interference inhibition, has become instrumental in the development of next-generation wireless systems. Important MIMO Technology that has emerged recently include Single-User MIMO, Multi-User MIMO, and Cooperative Relay (a method that allow base stations and relay stations to forward signals opportunistically) (Li et al., 2009). MIMO antenna technology can be utilised in cognitive radio networks to achieve, even under stringent interference-power constraints, substantial capacity gains for the secondary transmission (CR transmissions), by employing multi-antennas at the secondary transmitter; Wang, Liu, and Zhou (2009) have shown that the effect of interference diversity can be exploited in CR networks.

COGNITIVE RADIO TECHNOLOGY: Cognitive Radio (CR) networks bring into focus a new way of looking at wireless communication networks. Given the fact that the known frequency spectrum is dwindling rapidly, the need to efficiently utilize this very limited and expensive resource becomes more urgent. In Zimbabwe, the usage of radio spectrum resources and the regulation of radio emissions are functions of the Post and Telecommunications Regulatory Authority of Zimbabwe (POTRAZ) which is responsible for assigning spectrum to specific licence holders in specific geographic areas, in accordance with the static spectrum assignment policies. It was observed that spectral assignments by this method leave large portions of the assigned spectrum underutilized (Liang, Hoang, \& Chen, 2008). Therefore, within a TV licensed spectrum, some TV channels are heavily used at certain times, while others are largely unoccupied most of time. The unoccupied channels are referred to as voids which could become available opportunistically for other uses. Cognitive radios as defined by Dubey and Sharma (2010) and Devroye and Vahid (2008) will intelligently identify portions of the spectrum that are idle at a specific time and/or location, leading to the best spectrum and appropriate operating parameters being selected. It is this characteristic which is referred to as re-configurability (Wang \& Liu, 2011) that is utilised in WRAN for the opportunistic access of the TV frequencies for connectivity in remote and rural areas (Liang, Hoang, \& Chen, 2008). IEEE 802.22 standard is preferred for two main reasons: (a) no dedicated spectrum is required, hence service providers can save on spectrum license costs, and (b) a wide coverage area; a radius of $33 \mathrm{~km}$ that can be increased to $100 \mathrm{~km}$ (Liang, Hoang, \& Chen, 2008; Lee \& Akyldiz, 2011). This is essential in order to reach a large customer base in rural areas. The System architecture of WRAN is a simple point to multipoint system in which a WRAN base station (WRAN BS) serve multiple fixed-location wireless customer premise equipment (CPE) and an associated TV station. Equipped with a directional antenna for 
communication with BS and an omni-directional antenna to sense and measure the wireless environmental condition, the CPE is capable of adapting and to timely change the transmission characteristics as necessary in the face of primary user activities.

\section{The Proposal and Network Structure}

In this project, a comparison is performed based on basic requirements and technology parameters. Table 1 shows current technologies in use by Internet Service Providers (ISP) in Zimbabwe versus WRAN and Table 2 shows comparison of different technologies, in favour of WRAN technology. Figure 1 below was used as a typical structure for the proposed WRAN network for the Zimbabwean rural areas. The ZBC TV repeater/booster station shown in Figure 1, with its TV protection contour, is over $96 \mathrm{~km}$ away from Guruve Centre. The main ZBC TV transmitter is located in Harare. Possible cognitive radio users (or CPE) would be located at school premises such as St Francis and St Clair High school, shops and individual homesteads. The design would take into consideration the television protection contour and protection areas for other receivers including microphone systems as described in (Dubey \& Sharma, 2010; Liang, Hoang, \& Chen, 2008). Both infrastructure and ad hoc networks would be possible. Assuming a100square kilometre area around Guruve growth point, and considering a circular cell with a WRAN base station at the centre, the radius (R) of such a cell would be given as:

$$
\mathrm{R}=\sqrt{ }(100 / \pi) \mathrm{km} \text {, Hence, } \mathrm{R}=5.6 \mathrm{~km}
$$

Table 1. Technology choice-comparison of basic requirements and technology parameters

\begin{tabular}{lcccc}
\hline \multirow{2}{*}{ Parameters } & \multicolumn{4}{c}{ Internet Service Providers } \\
\cline { 2 - 5 } & Econet Wireless & $\begin{array}{c}\text { Telecel } \\
\text { Zimbabwe }\end{array}$ & NetOne Zimbabwe & Project Proposal (WRAN) \\
\hline Network Type & Cellular & Cellular & Cellular & Cognitive Radio (WRAN) \\
Industrial Standard & $\begin{array}{c}\text { 3G (WCDMA) \& } \\
\text { WiMAX }\end{array}$ & $2 \mathrm{G}(\mathrm{GSM})$ & $\begin{array}{c}3 \mathrm{G} \text { (WCDMA) } \\
2 \mathrm{G}(\mathrm{GSM})\end{array}$ & IEEE 802.22 \\
$\begin{array}{l}\text { Maximum Data } \\
\text { Rate }\end{array}$ & Up to $30 \mathrm{Mb} / \mathrm{s}$ & $2 \mathrm{Mb} / \mathrm{s}$ & Up to $14 \mathrm{Mb} / \mathrm{s}$ & $31 \mathrm{Mb} / \mathrm{s}$ \\
$\begin{array}{l}\text { Channel bandwidth } \\
\text { Large Coverage }\end{array}$ & $5 \mathrm{MHz} \& 20 \mathrm{MHz}$ & $200 \mathrm{kHz}$ & $200 \mathrm{kHz} \& 5 \mathrm{MHz}$ & $6 \mathrm{MHz}, 7 \mathrm{MHz}$ or $8 \mathrm{MHz}$ \\
$\begin{array}{l}\text { Range } \\
\text { Broadband } \\
\text { availability }\end{array}$ & Yes & No & No & Yes \\
$\begin{array}{l}\text { Fast Deployment } \\
\text { Acceptable Cost }\end{array}$ & Yes & $(0.2$ to $2 \mathrm{~km})$ & $(1$ to $2 \mathrm{~km})$ & $(30$ to $100 \mathrm{~km})$ \\
\hline
\end{tabular}

Table 2. Comparison of different technologies

\begin{tabular}{cccc}
\hline \multirow{2}{*}{ Parameters } & \multicolumn{3}{c}{ Technology } \\
\cline { 2 - 4 } & WIMAX & Mobile Cellular & WRAN Project \\
\hline Coverage range (Cell radius) & $4 \mathrm{~km}$ & $3 \mathrm{~km}$ & $5.6 \mathrm{~km}$ \\
No. of cells per $100 \mathrm{~km}^{2}$ & 2 & 3 & 1 \\
Total channel capacity per cell & $30 \mathrm{Mbps}$ & $5 \mathrm{Mbps}$ & $72 \mathrm{Mbps}$ \\
Subscribers per uplink channel & 78 & 13 & 187 \\
Subscribers per downlink channel & 20 & 3 & 48 \\
\hline
\end{tabular}

Assumptions for Table 2: System capacity per user, DL $=1.5 \mathrm{Mbps}$; System Capacity per user, UL $=384 \mathrm{kbps}$; Total Available Spectrum = $24 \mathrm{MHz}$; Circular Cell type was used. 


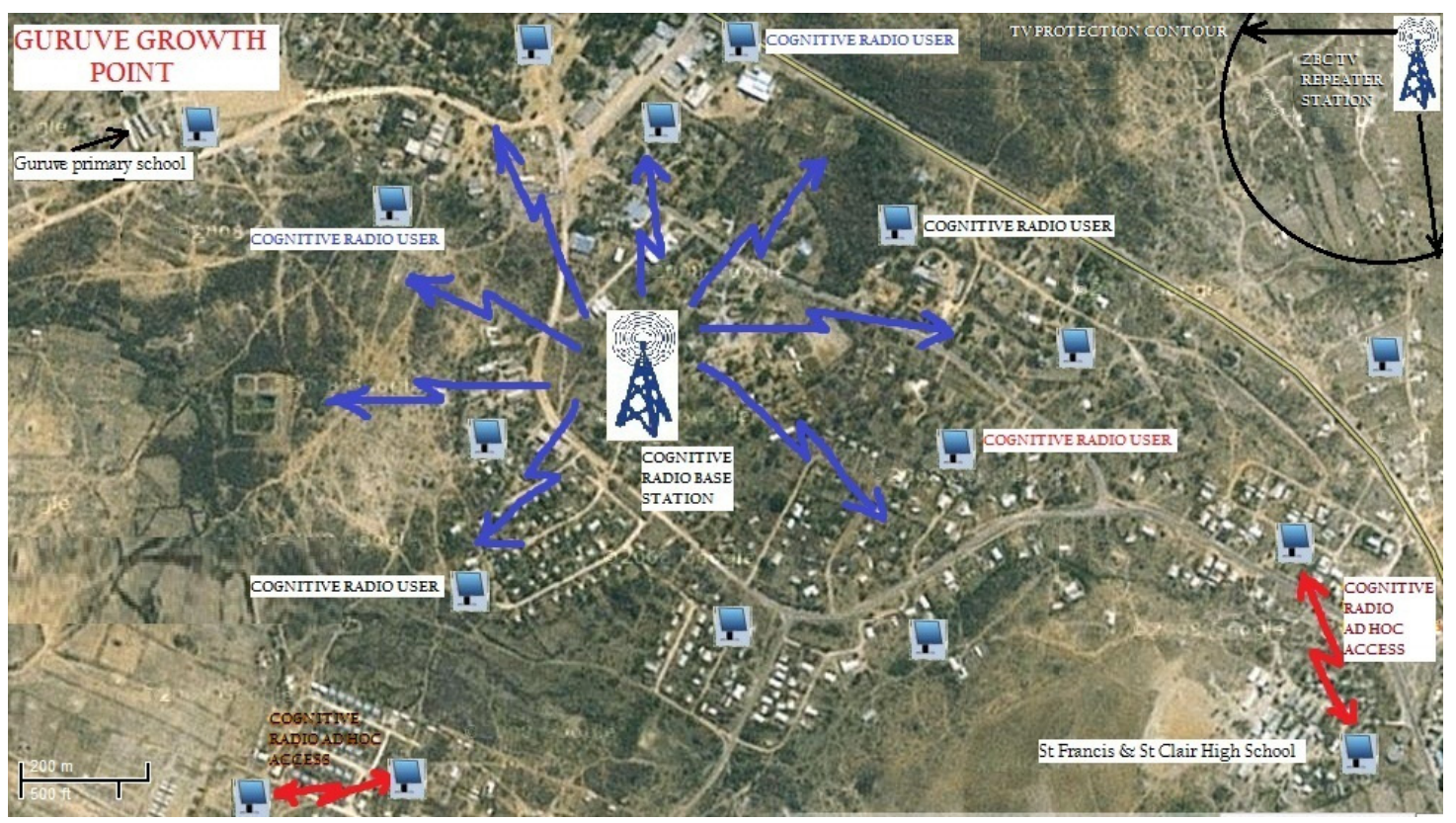

Figure 1. Proposed WREN configuration for guruve growth point

Only one WRAN base station would be required to service Guruve growth point. For the homesteads outside the $5.6 \mathrm{~km}$ radius and do not have line of sight with the base station, WRAN ad hoc network would be preferred although they will still be within WRAN's average coverage radius of $33 \mathrm{~km}$. A similar design proposal could be made for remote areas.

\section{Methodology of Simulation}

Simulating the above is obviously very difficult as there are many unknown variables such as the ZBC TV repeater/booster station transmitter power, total number of channels or frequency bands, actual environmental topography, sources of interference, number of prospective users etc. Hence the prime objective of this simulation is to show the possibility of harnessing idle ZBC TV channels/frequency bands using CR technology. In this methodology of simulation, we set four ZBC TV channels operating to be within $50 \mathrm{MHz}$ to $862 \mathrm{MHz}$ frequency band, namely: ZBC TV1, ZBC TV2, ZBC TV3 and ZBC TV4 at carrier frequencies $50 \mathrm{MHz}, 60 \mathrm{MHz}$, $70 \mathrm{MHz}$ and $80 \mathrm{MHz}$ respectively. Some of the TV channels will be assumed active with user data, while others will be without user data information, thus referred to as idle or spectrum voids as shown in Figure 2 below. The active channels are assumed allocated to primary users while secondary users opportunistically utilise the inactive spectrum voids. Zimbabwe like many other developing countries does not have 24 hours TV transmission but transmits between 1400 and 2300 hours; which leaves TV channels idle for the rest of the day. It is therefore assumed in this simulation that carrier frequencies operating within this time slot will be active; any other combination will be assumed inactive or idle.
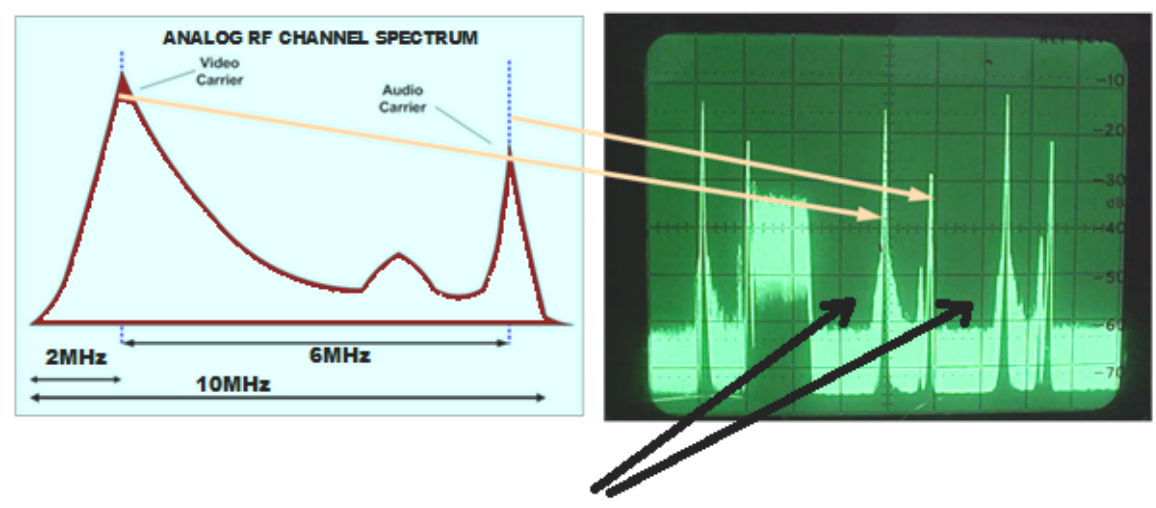

spectrum voids/holes

Figure 2. Typical Analog channel RF spectrum 
The cognitive radio base station in this project plays a simple role of coordinating the secondary users with minimum sensing abilities. Next, an energy detection type of sensing such as power spectral density estimation (PSD) graph is employed in this project. Other sensing methods such as waveform based sensing, cyclostationary-based sensing, matched filter sensing etc. are more complex compared to the energy detection.

In the spectral density estimation, the power carried by a wave per unit frequency of the signal can be found by the area under the PSD curve of the signal. Then, the noise power (or noise floor level) of the channels was used to determine a noise free threshold of the channels which was estimated at $-10 \mathrm{~dB} / \mathrm{Hz}$.

In this simulation, it was assumed that if the total measured energy on a channel such as the power spectral density was below this predetermined threshold then that channel would be taken as available or inactive. Then, the resultant PSD graphs in the simulation enabled us to observe these signal power changes in the channel as its status changed from idle to being active indicating CR secondary user opportunistic activity. PSD though simple, has some drawback, such as it is not able to differentiate interference from primary users and noise. Hence it displays poor performance under low signal-to-noise ratio (SNR) values since the threshold depends on noise floor level. The simulation technique used in this project was adopted from http://intcube.com/edu/projects/codes/crad.m and had been improved and proposed a new method to reflect typical analog TV bands. MATLAB 7.0.4 R14 SP2 was used for the simulation in this project. The summary of the methodology of simulation are as follow:

1) Set four ZBC TV channels operating within the $50 \mathrm{MHz}$ to $862 \mathrm{MHz}$ frequency band, namely: ZBC TV1, ZBC TV2, ZBC TV3 and ZBC TV4 at carrier frequencies $50 \mathrm{MHz}, 60 \mathrm{MHz}, 70 \mathrm{MHz}$ and 80 $\mathrm{MHz}$ respectively.

2) Activate the carrier frequencies operation, while any other combination will be assumed inactive or idle.

3) Perform the graph for energy detection type of sensing such as power spectral density estimation (PSD).

4) Determine the power carried by a wave per unit frequency of the signal from the area under the PSD curve of the signal.

5) Determine the noise free threshold of the channels from the noise power or noise floor level.

6) Observe the signal power changes in the channel from the PSD graphs.

7) Determine the status from the changes of idle to being active in the CR secondary user opportunistic activity.

The simulation was run in accordance with the flow chart shown below in Figure 3. 


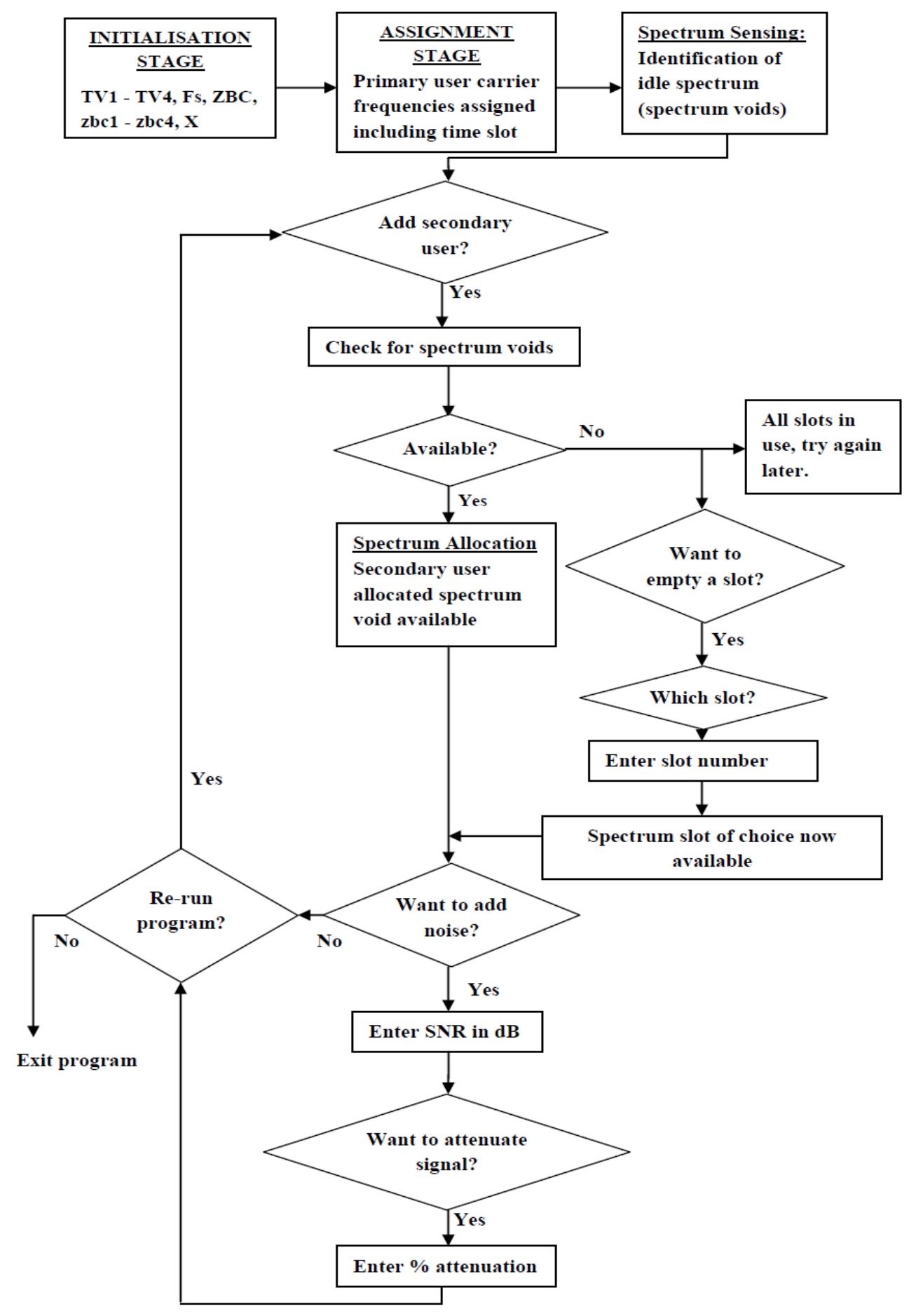

Figure 3. Simulation flow chart

\section{Results and Discussion}

The results obtained after running the simulation could be categorised into three main stages with each stage depicting a cognitive radio process. PSD estimation graphs were used to represent the results in each stage. The first stage results, shown in Figure 4 below, depicted the allocation of primary user carrier channels. This process is usually done at the transmitter station where data signals are modulated transmitted and the time slot for broadcasting set. The interrogation and answer box of Figure 4 showed that entering a YES for a primary user and a YES for time slot was synonymous to allocating a carrier frequency to a primary user for the duration of time stipulated by the time slot and a NO or a combination of NO and YES, indicated a spectrum void/idle 
channel which could be used by a secondary user. In our case, the primary users were located at $50 \mathrm{MHz}$ and 70 $\mathrm{MHz}$ while voids were at $60 \mathrm{MHz}$ and $80 \mathrm{MHz}$ respectively as shown in Figure 4 below.

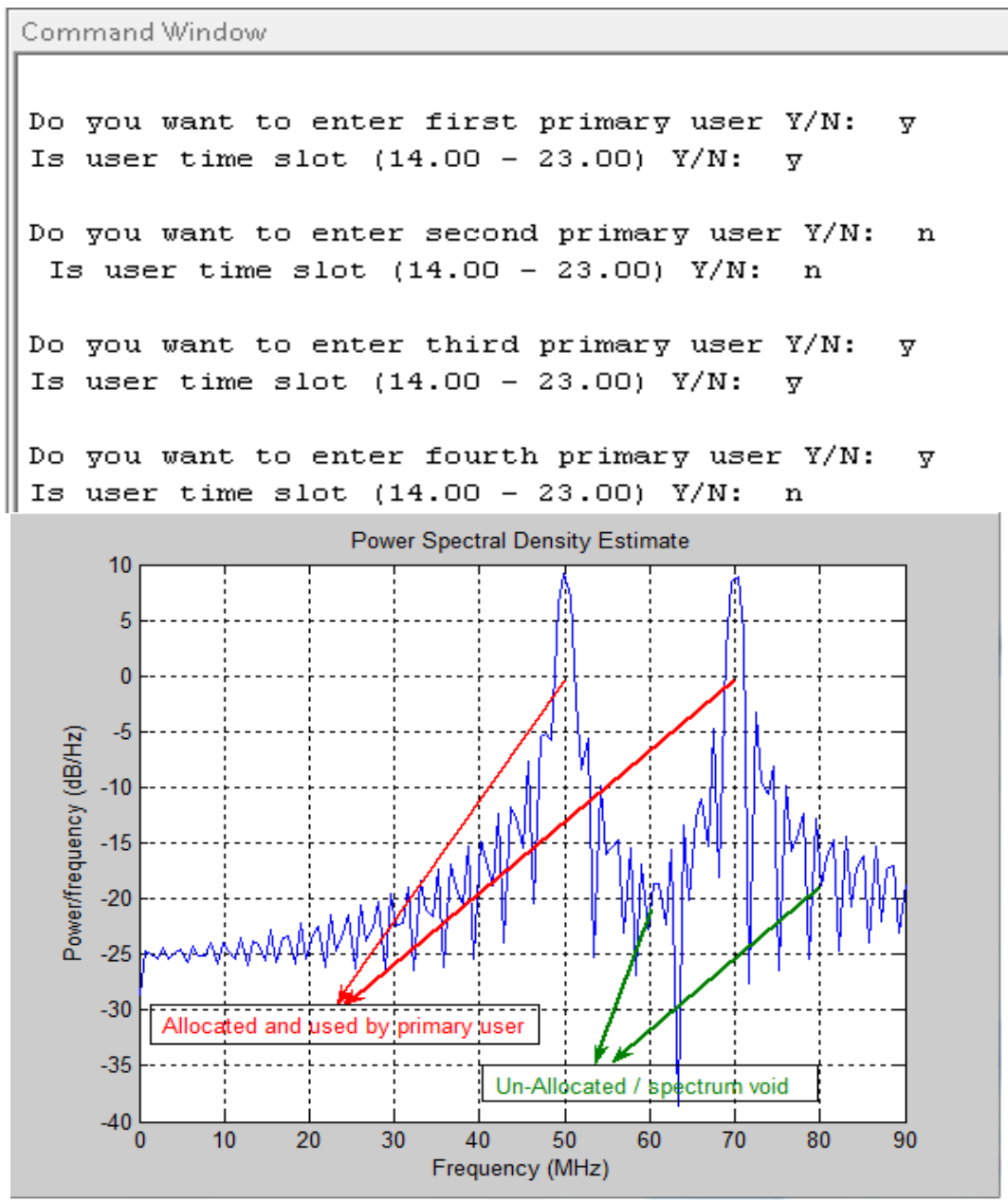

Figure 4. Spectrum Sensing-Identification of idle spectrum (voids)

The first stage was flexible and could be used to indicate presence of primary users and at the same time create spectrum voids at any of the designated frequencies. We observed from Figure 4 that the PSD peaked above the threshold power $-10 \mathrm{~dB} / \mathrm{Hz}$ at active frequency bands. All other frequency bands with PSDs below this threshold were taken as idle. The PSDs of the active bands have a SINC function shape which is typical of a rectangular window function used in computing the PSD; the side-lobes constituting unwanted signal/noise. The unwanted signal and noise usually determine the threshold value of the PSD. One can therefore relate Figure 4 to a spectrum that has idle channels which must be sensed and utilised by a cognitive radio device. It was also observed from Figure 4 that, counting from left to right, the first and third frequency slots that is TV1 (50 MHz) and TV3 $(70 \mathrm{MHz})$ respectively were occupied by primary users while the second and fourth slots TV2 (60 MHz) and TV4 $(80 \mathrm{MHz})$ respectively were voids. The second stage of the simulation dealt with the sensing and allocation of secondary users as is shown in Figure 5 above. The secondary users would opportunistically try to occupy the second and fourth slots which are available and thus help improve spectral efficiency. The last two lines of the dialogue box of Figure 5, below, indicated secondary users desiring to enter the spectrum and occupy these voids. A YES in this case activated the second slot as shown in Figure 5 (the graph portion); thus the PSD of that channel increased and became more than the threshold, indicating activity as evidenced by in the graph by the appearance of the middle PSD in Figure 5 while the carrier at $80 \mathrm{MHz}$ still remained unoccupied. It is important to note that the sensing and allocation stages occur in an environment that is prone to all kinds of noise and interference hence for the purposes of this project, the inclusion of noise and attenuation processes as shown in Figures 6 and 7 below will help create such an environment. 

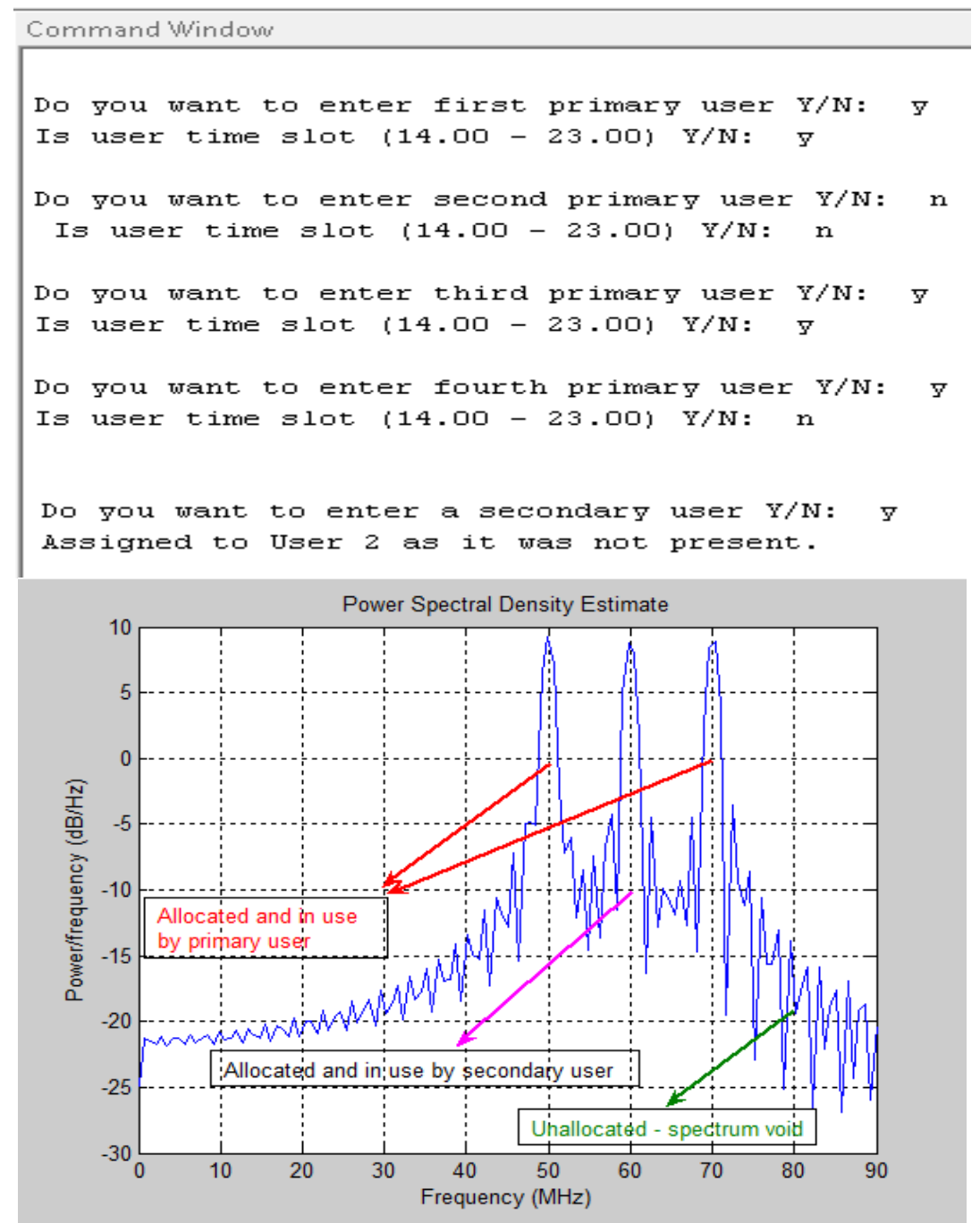

Figure 5. Spectrum Allocation-Secondary user allocated first spectrum void

The presence of noise was simulated here by the introduction of Additive white Gaussian noise (AWGN) into the channel. Figure 6 shows the effect of adding $10 \mathrm{~dB}$ of AWGN. It was observed that after the introduction of noise, Figure 6 could be divided into three distinct areas. The left side of the graph showed the noisy signal. The idle part showed the active channels in a noisy environment. The introduction of AWGN had the effect of raising the noise power level of the system to above $-10 \mathrm{~dB} / \mathrm{Hz}$ such that the PSD of the noise in the vicinity of the void (i.e. at $80 \mathrm{MHz}$ ) was computed to be higher than the threshold setting. This then gave rise to errors in the interpretation of the presence of either primary or secondary users in the system. A false signal that indicated that the idle channel was now allocated to a secondary or primary user was received. The dialogue box of Figure 6 after adding noise showed all four channels being active affirming the fact that PSD sensing is not able to differentiate interference from primary users and noise. 


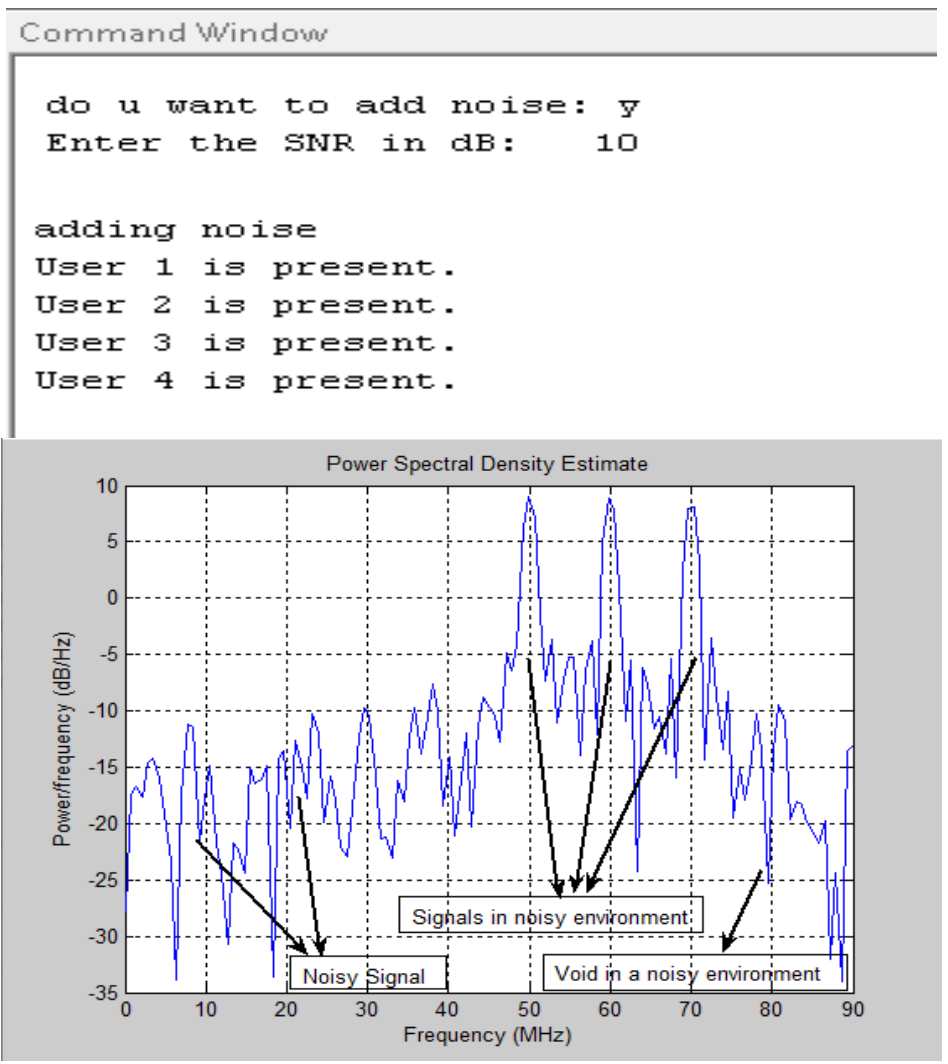

Figure 6. Spectrum Allocation-Example of a noisy channel

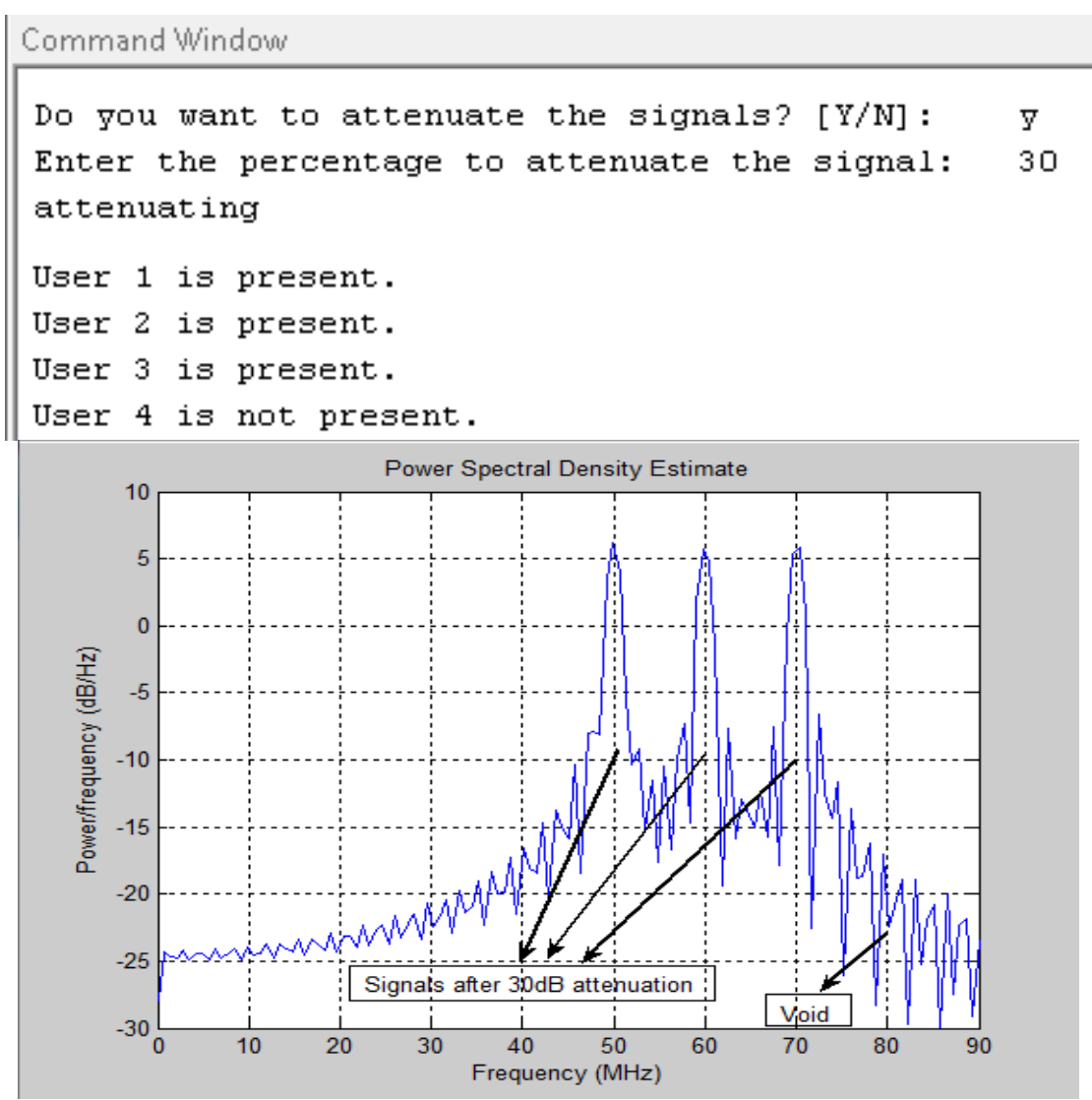

Figure 7. Spectrum Allocation-Example of a noise attenuated channel 
In Figure 7, the reverse of Figure 6 is shown in which $30 \mathrm{~dB}$ attenuation was applied to the noisy system, with the result showing the true state of the channel at that moment; that is inactive as can be seen from Figure 8 dialogue box. Trying to identify or sense the presence of primary users or other secondary users in low signal environments resulting from noisy environments, interference, hidden terminal situations etc. is one of the many challenges of this technology. The result shown in Figure 8 takes us back to the secondary user trying to access the idle channel. The same process that was described before will be repeated rendering the channel active; hence the fourth PSD becomes higher than the threshold power. The appearance of the fourth PSD seen in the graph, signify the presence of activity in the channel. The third stage of the simulation dealt with a situation in which a primary user tries to access a channel previously allocated to it but for some reasons, e.g. a technical fault the primary user had not utilised the channel. During that outage the channel would be sensed as idle resulting in a secondary user opportunistically accessing and utilising the channel. As soon as the fault is rectified, the primary user would require usage of the same portion of spectrum. This scenario, which is typical of TV channels, and is similar to spectrum mobility, would lead to "the emptying of slot" process described in this simulation; Figure 9 below shows the results of such a process. This process is synonymous to a secondary user vacating the channel should a primary user activity require usage of the same portion of spectrum. Hence it may be necessary that the secondary user to relocate to another vacant portion of the spectrum in order to continue in communication. Two things became clear in Figure 9 below. Firstly, once all the channels are active any attempts to allocate an extra secondary user would not be possible. This result was expected; additional users would be dropped once the network was busy.

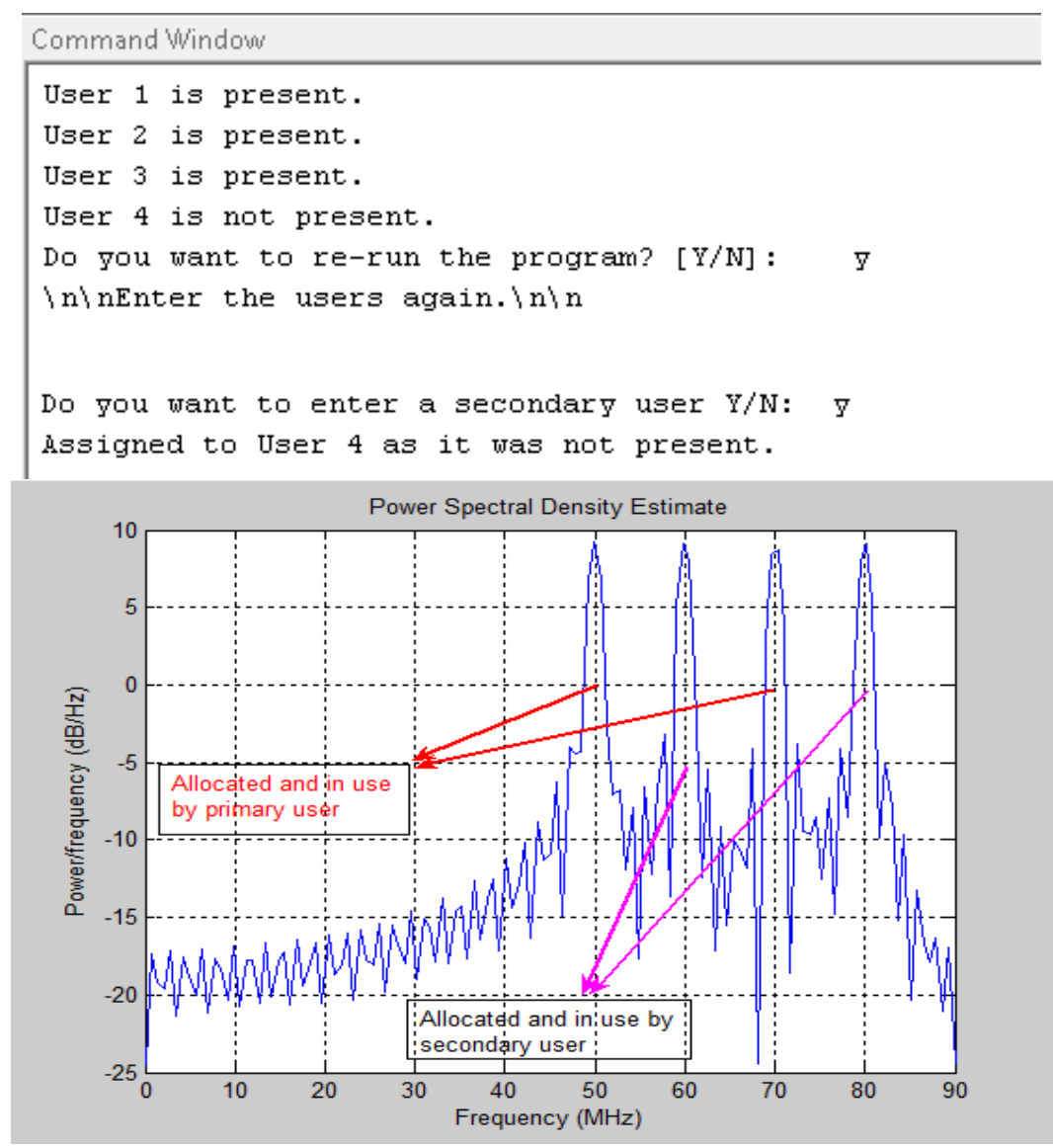

Figure 8. Spectrum Allocation-Secondary user allocated 2nd spectrum void

Secondly, a slot must be made available should a primary user be detected near a secondary user and requires use of the same portion of spectrum occupied by the secondary user; primary user has priority. This is shown in Figures 9 and 10 where the secondary user vacates (Figure 9) the frequency band to make way for the primary user (Figure 10). This process of emptying a slot relates to and resembles spectrum mobility in cognitive radio networks. The whole process of sensing, allocating, and emptying of slot was then repeated as long as spectrum voids existed. The result of this simulation has shown that it is possible to use cognitive radio technology to 
access and utilise ZBC TV's idle channels since the basics of cognitive radio technology (WRAN) has been successfully simulated in this project. However it is a known fact that full cognitive radio implementation is not currently realiable due to lack of truly cognitive radio devices; but the above result points in the right direction that the possibility of success using basic intelligent reconfigurable cognitive radio to effect cognitive radio network technology.

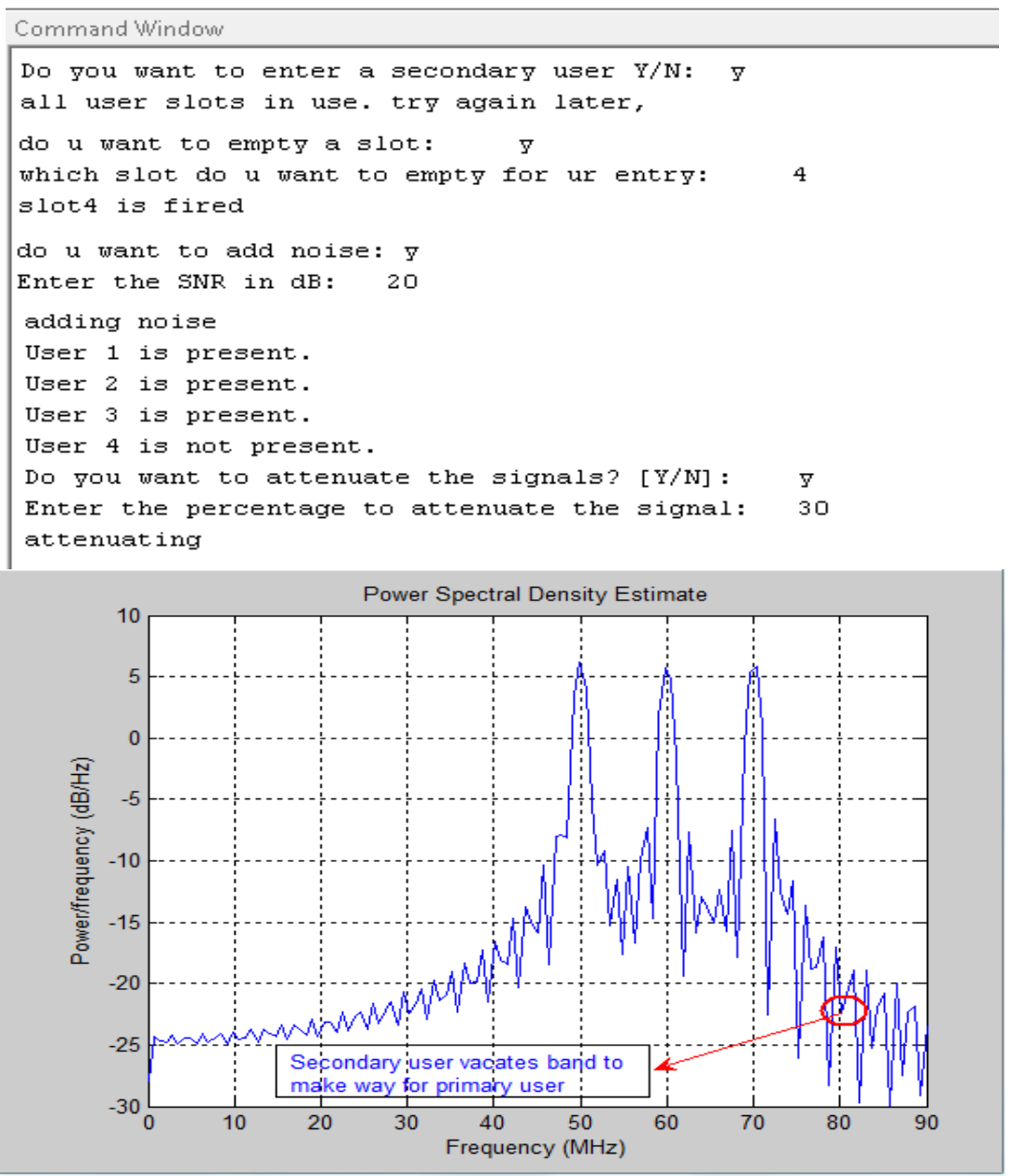

Figure 9. Spectrum Allocation-Secondary user making way for primary user

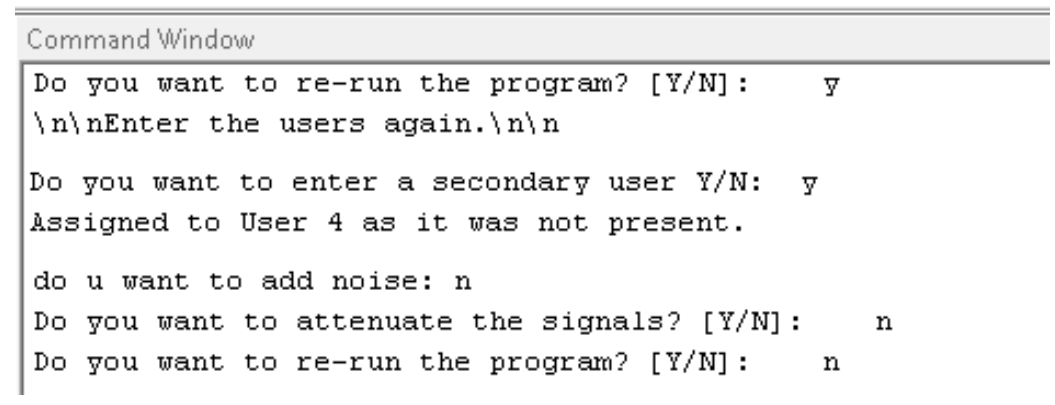




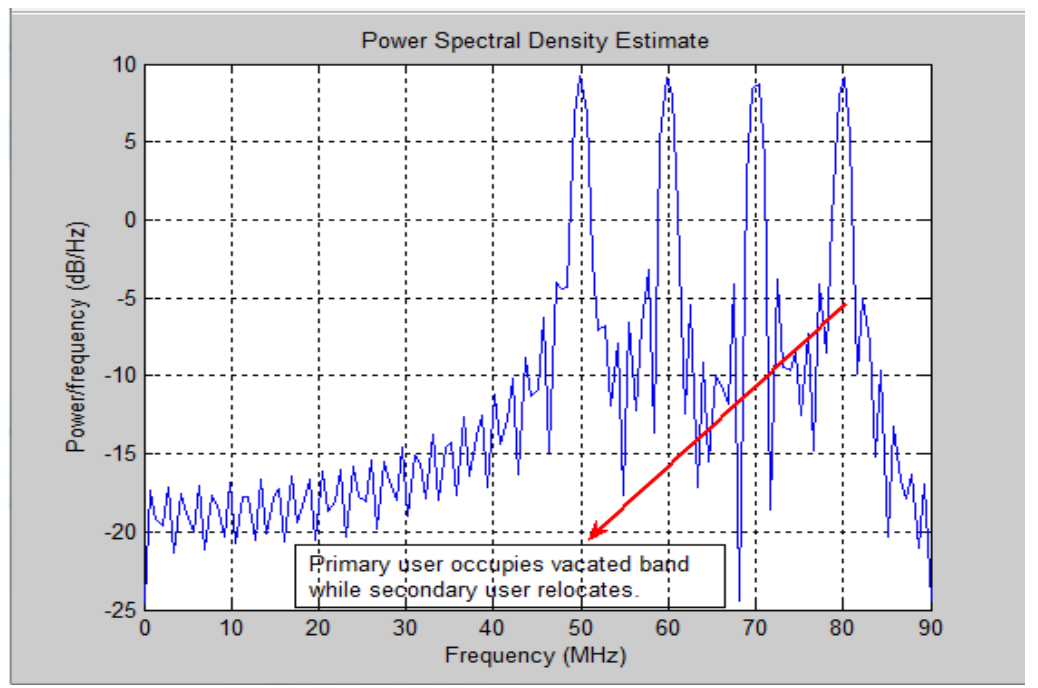

Figure 10. Spectrum Allocation: Example: primary user occupying vacated spectrum

\section{Implementation Challenges}

The onsite implementation of the above project will not be easy but will obviously have a number of challenges. The challenges could be grouped into two categories, which are economic and technical challenges. The economic challenges will involve: first, persuading service providers to invest in this new technology (CR network) and particularly to invest in a low dividend market and secondly, trying to bring about a paradigm shift within the rural community. In other words, convincing the rural market to embrace this new technology rather than stick to the more traditional ways of just watching television programs, listening to radio broadcast programs and use the telephone for voice communications. However the younger generation might be easier to convince as they are more exposed to new and upcoming technologies. The technical challenges include, conducting real time simulations based on the above design that incorporates onsite testing and identification of physical and environmental requirements, coverage and outage criteria etc. Due to the simplicity of the proposed CR structure and also because that full cognitive radios are nonexistent at the moment, incorporating MIMO technology at this stage involving implementation, simulation and associated effects proved challenging. Hence no work was done in this project that incorporated MIMO effects on the CR networks. This method, though not new to the communications industry would have required the recalculation of the contribution of WRAN base station interference on the television booster station and the associated television booster station protection contour, since the television booster power would be less than the original television transmitter power in Harare. Field measurements usually impose equipment errors onto the simulated results hence it will be difficult to accurately determine whether a channel is occupied. Antenna height at both base station and customer premises would also pose another reasonable challenge - the calculation of which will depend on the surrounding topography. Normally antennas for the reception of TV channels are very tall and hence are prone to lighting strikes; Zimbabwe being in the tropical region experiences frequent thunder and lightning activities. Thus the rate of outages might become worrisome. The issue of the hidden terminal will obviously play an important role in this method. The most important challenge would be the calculation and mitigation of interference contributed by distant television stations, other WRAN networks, micro-cellular networks etc. The resultant interference emanating from the overlapping nodes is a major cause of concern and is usually referred to as the coexistence problem.

\section{Conclusion and Future Studies}

Based on the foregoing discussions, the aims of the project, literature reviews on cognitive radio (including WRAN) and the fact that ZBC's TV bands are largely underutilised, we have proposed an algorithm and contribute towards WRAN of IEEE 802.2, and it shows very promising candidate technology for providing broadband wireless access to rural Areas in Zimbabwe. The Zimbabwean problem was expounded and possible solutions of bringing broadband internet connectivity to the rural community sought by examining trends in other countries with similar problems and reviewing new technologies such as cognitive radio. It was therefore shown that WRAN can utilise opportunistically TV broadcast bands and exhibiting large coverage range and favourable per cell capacity, was identified as a suitable broadband access for these sparsely populated areas that 
are difficult to economically service by either means of wireless or wired. The simulation has been performed by using Matlab software, which is the most suitable tool in engineering field, especially in the field of wireless communication. The cognitive radio network which is a latest and interested field, has been adopted and some parameters have been tested in this project and it was successfully carried out on VHF TV bands shows a good finding. The simulation revealed that it was possible to harness idle ZBC TV channels/frequency bands using cognitive radio technology. Implementation challenges which included both technical and economy were noted and enunciated. Major challenges highlighted in this project still require further investigations include: the effect that the antenna height has on the hidden terminal problem, and the way MIMO could be incorporated to mitigate such effects in WRAN networks; and the calculation and mitigation of interference contribution from distant television stations, WRAN networks in the vicinity and other primary stations and users that coexist with the problem.

\section{References}

http://intcube.com/edu/projects/codes/crad.m

http://www.techzim.co.zw/2010/10/no-real-broadband-yet-in-zimbabwe/

http://www.thestandard.co.zw/local/28597-zbc-tv-viewership-hits-rock-bottom.html

Bauch, G., \& Alexiou, A. (2008). MIMO Technologies for the Wireless Future. Personal, indoor and mobile Radio Communications, 2008, PIMRC 2008. IEEE $19^{\text {th }}$ International Symposium, 1-6. http://dx.doi.org/10.1109/PIMRC.2008.4699969

Devroye, M. V., \& Vahid, T. (2008). Cognitive Radio Networks'. Signal Processing, IEEE., 25(6), 12-23. http://dx.doi.org/10.1109/MSP.2008.929286

Dubey, R., \& Sharma, S. (2010). Distributed Shared Spectrum Techniques for Cognitive Wireless Radio Networks. Computational Intelligence and Communication Networks (CICN), International Conference, 259-264.

International Telecommunication Union. (2010). Information Society Statistical Profiles 2009 Africa. Retrieved from http://www.itu.int/ITU-D/ict/material/ISSP09-AFR_final-en.pdf

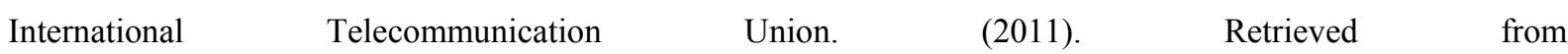
http://www.itu.int/ITU-D/ict/publications/idi/2009/material/IDI2009_w5.pdf

Lee, W. Y., \& Akyldiz, I. F. (2011). A Spectrum Decision Framework for Cognitive radio network. Mobile Computing IEEE Transactions, 10(2), 161-174. http://dx.doi.org/10.1109/TMC.2010.147

Liang, Y. C., Hoang, A. T., \& Chen, H. H. (2008). Cognitive radio on TV bands: a new approach to provide wireless connectivity for rural areas. Wireless Communications, IEEE, 15(3). $16-22$. http://dx.doi.org/10.1109/MWC.2008.4547518

Li, Q. H., Lin, E. X. T., Zhang, J. Z., \& Wonil, R. (2009). Advancement of MIMO Technology in WiMax-from IEEE802.16d-e-j to 802.16m. Communications Magazine, IEEE., 47(6), 100-107.

Wang, B. B., \& Liu, K. J. R. (2011). Advances in Cognitive Radio Networks: A Survey. Selected Topics in Signal Processing, IEEE Journal, 5(1), 5-23. http://dx.doi.org/10.1109/JSTSP.2010.2093210

Wang, S. B., Liu, Q., \& Zhou, Z. (2009). A Cooperative Cognitive Radio Network Using Smart Antenna. Communications Technology and Applications, ICCTA'09, IEEE International Conference, 450-456. http://dx.doi.org/10.1109/ICCOMTA.2009.5349161

\section{Copyrights}

Copyright for this article is retained by the author(s), with first publication rights granted to the journal.

This is an open-access article distributed under the terms and conditions of the Creative Commons Attribution license (http://creativecommons.org/licenses/by/3.0/). 